\title{
Concomitant Medication Use Sponsor Defined Identifier
}

National Cancer Institute

\section{Source}

National Cancer Institute. Concomitant Medication Use Sponsor Defined Identifier. NCI

Thesaurus. Code C83075.

One or more sponsor-defined characters used to identify, name, or characterize the concomitant medication. 